$\$$ Research Square

\title{
Psychometric validation of the Fear of Progression Questionnaire- Short Form in acute pancreatitis patients (AP-FoP-Q-SF)
}

\author{
Shu-Li Ma \\ Affiliated Hospital of Nantong University \\ Xiao-Xi Yang \\ Northern Jiangsu People's Hospital \\ Sheng-Xiao Xiang \\ Yangzhou University \\ Guo-Tao Lu \\ Yangzhou University \\ Wei-Juan Gong \\ Yangzhou University \\ Weiwei Chen ( $\nabla$ cww1984@126.com ) \\ Northern Jiangsu People's Hospital
}

\section{Research Article}

Keywords: acute pancreatitis, fear of progression, validation study

Posted Date: February 3rd, 2022

DOI: https://doi.org/10.21203/rs.3.rs-1206739/v1

License: (c) (i) This work is licensed under a Creative Commons Attribution 4.0 International License. Read Full License 


\section{Abstract}

Background: Fear of progression (FoP) is present in acute pancreatitis (AP) patients and has an impact on quality of life and selfmanagement, but lack of assessment tools. This study therefore aimed to develop and evaluates the psychometric properties of the fear of Progression Questionnaire-Short Form in acute pancreatitis patients (AP-FoP-Q-SF). Besides, the cut-off score for high FoP and identification of patient characteristics associated with FoP.

Methods: A prospective cross-sectional validation study was performed in 417 patients diagnosed with AP. Redundant items were systematically excluded. Internal consistency (Cronbach's a), factorial structure (principal component analysis), convergent validity (confirmatory factor analysis), and criterion validity tested against quality-of-life and anxiety were assessed. A receiver operating characteristic (ROC) curve analysis was performed to identify the most appropriate cutoff value for high FoP.

Associations between patient variables and FoP were evaluated using multiple logistic regression.

Results: The AP-FoP-Q-SF based on the FoP-Q-SF were developed. Three items were excluded using item analysis. Exploratory and confirmatory factor analyses revealed a clear two-factor structure ( $57.4 \%$ explained variance). Internal consistency was acceptable (Cronbach's a 0.771 ). The cutoff of 26 identified $35.3 \%$ of patients with high FoP scores. High FoP scores were associated with age $(\mathrm{OR}=0.96,95 \% \mathrm{Cl}: 0.94-0.98)$, recurrence times $(\mathrm{OR}=1.22,95 \% \mathrm{Cl}: 1.02-1.45)$ and anxiety $(\mathrm{OR}=1.27,95 \% \mathrm{Cl}: 1.16-1.40)$.

Conclusion: The AP-FoP-Q-SF is a good candidate for use as a screening tool for FoP in AP patients. Clinicians can use the AP-FoP-Q-SF to assess this kind of emotion and take promotion programs to avoid worse effects.

\section{Background}

The etiology of acute pancreatitis (AP) is complex; the course of disease is unpredictable and progressive, and the relapses are common[1]. In addition, recurrent episodes of acute pancreatitis can progress to chronic pancreatitis, which can significantly reduce patients' quality of life and is associated with pancreatic cancer[2]. These increase fear in patients. Fear is a negative emotion, which reduces the patient's pain threshold, sleep quality, increases the adverse reaction of the digestive system, and takes actions that are not conducive to health[3]. Negative emotion is associated with longer hospital stays, higher hospital costs, and readmission[4, 5]. Meanwhile, the lack of ability to self-manage the disease and decreased quality of life of patients with AP are associated with fear of future health[6].

Fear of future health has been found in patients with AP, acute recurrent pancreatitis and chronic pancreatitis[2, 6, 7]. Fear in patients with AP is specific and important. However, the psychological problem of fear of disease progression is often underestimated or unrecognized. One reason is the lack of assessment tools, which makes fear of disease progression difficult to assess comprehensively.

In 2003, Professor Dankert proposed the concept of fear of progression (FoP), which is defined as fear of all the biopsychosocial consequences of disease progression or fear of illness recurrence[8]. The most common tools used to assess FoP are the Fear of Progression Questionnaire[9], Fear of Cancer Recurrence Inventory[10] and Fear of Progression Questionnaire-Short Form (FoP-Q-SF)[11]. They are used in chronic diseases, and none, to our knowledge, specifically assess FoP in AP patients. The FoP-Q-SF has been validated in a variety of diseases, and qualitative studies reported that the fear content of patients with AP was similar to the items in the FoP-Q-SF.

Therefore, the purpose of this study was to further develop and improve the content of the questionnaire according to the fear of disease progression in patients with AP and verify its reliability and validity.

\section{Methods}

The development, validation, and reliability testing of the AP FoP-Q-SF (AP-FoP-Q-SF) were performed in a prospective cross-sectional study.

\section{Participants}

A convenience sample of 417 participants was recruited from August 2020 to February 2021 in the gastroenterology departments of three tertiary hospitals in the eastern region of China. The inclusion criteria for inpatients were as follows: (a) diagnosis of AP were based on the 2012 revised Atlanta criteria[12]; (b) Patients aged $\geq 18$ years and < 80 years; and (c) agreement to participate in the study. The exclusion criteria were as follows: (a) diagnostic suspicion or a definitive diagnosis of severe illness, such as cancer or chronic failure of the heart, liver or other major organs; (b) pregnancy or breastfeeding; or (c) cognitive impairment or a history of psychiatric illness.

Patients completed the questionnaire after informed consent was obtained, and the completeness of the questionnaire was checked by the researcher after the patient finished. Patients with incomplete questionnaires were asked to complete the questionnaire on site. For sentences 
that were difficult to understand, standard guidelines were used to assist the patient.

\section{Procedures}

\section{Preliminary Adjustment of Questionnaire Items}

The FoP-Q-SF is a multidimensional self-report questionnaire comprising 12 items belonging to four categories (affective reactions, partnership/family issues, occupation, and loss of autonomy)[13]. Five Likert-style items were used to assess the degree of FoP (ranging from 1 ("never") to 5 ("very often")). In qualitative studies, FoP and special treatments, worry about work and the burden on family members were often mentioned by patients with AP[7, 14]. The items of the questionnaire and the results of qualitative studies were discussed among an internal expert group consisting of 3 pancreatic disease experts, 1 psychological expert and 1 scale expert, and the items and expressions of the scale were finally determined by consensus.

\section{Determination of Questionnaire Items}

To develop the AP-FoP-Q-SF, an item analysis comprising the following six guidelines was conducted:

1. If the distribution of answers revealed floor or ceiling effects, i.e., a value of 1 or 5 accounted for more than $50 \%$ of the responses, respectively, questions were excluded because of insufficient measurement precision[15].

2. If the answer distribution showed extreme skewness, the items with skewness coefficients > 2 were deleted[16].

3. If the commonalities between the items indicated that the item could explain the variability of the same psychological trait, principal component analysis was used to extract one common factor to test the commonness among the items. If the factor loadings were less than 0.5 , they were deleted[17].

4. If the Pearson's correlation coefficient between item scores and total scale scores was $\leq 0.50$, the item was deleted[18].

5. The $t$-value was calculated between the items of the upper $27 \%$ and lower $27 \%$ of cases to test the discriminability of the questionnaire. If the items showed poor discriminability $(\mathrm{t}<3)$, they were deleted.

6. Cronbach's alpha method was adopted by analyzing whether the Cronbach's alpha of the instrument increased after deleting the current item.

\section{Reliability and Validity Testing of the Questionnaire}

\section{Validation}

Three types of validation were investigated.

1. Content validity: The FOP-Q-SF is a simplified version of the FOP-Q, which has been widely used and verified in a variety of diseases, indicating that relevant experts affirm its content. In addition, the internal expert panel of this study also discussed the content of the questionnaire and ensured that all important aspects were covered in it through consensus.

2. Construct validity: Construct validity refers to the degree to which the questionnaire can measure a certain psychological trait. We used the first half of the data to conduct exploratory factor analysis (EFA) to explore the structure of the questionnaire. We then conducted confirmatory factor analysis (CFA) using the second half of the data to verify the factor structure and assess factor loadings and model fit.

3. Criterion validity: Based on previous studies[19], the Hospital Anxiety and Depression Scale (HADS) and the shortened version of the SF36 Health Survey (SF-12) were used to verify the criterion validity of the AP-FOP-Q-SF.

\section{Reliability}

To verify the stability and heterogeneity of the questionnaire results, the Cronbach's alpha and split-half reliability methods were adopted in this study.

\section{Statistical Analysis}

The number of participants with no missing demographic or clinical characteristics, a completed AP-FoP-Q-SF and available HADS and SF-12 results was small $(\mathrm{N}=416)$. This was managed using pairwise deletion. Descriptive statistics are used to describe the demographic and clinical characteristics. Frequency and percentages are used to summarize categorical variables, and the mean \pm standard deviation is used to summarize quantitative measures. 
To evaluate the construct validity of the AP-FoP-Q-SF, we randomly divided the sample into two datasets. The Kaiser-Meyer-Olkin test and Bartlett's test of sphericity were used to confirm the sample and item adequacy in one subsample of the two datasets. If the data were suitable for exploratory factor analysis (EFA), a principal component analysis with varimax rotation was used to assess the main factors. An eigenvalue $>1$ was used to determine the underlying factor structure. The other subsample was used for confirmatory factor analysis (CFA). The maximum-likelihood-robust estimation method was applied to estimate the fit of the underlying structure. The goodness-of-fit was verified by using the following indexes: (1) the relative chi-square $\left(\chi^{2} / d f<3\right)$, (2) the root mean square error of approximation (RMSEA<0.08), (3) the normed fit index (NFI>0.90), (4) the incremental fit index (IFI>0.90), (5) the Tucker-Lewis index (TLI>0.90) and (6) the comparative fit index (CFI>0.90)[20]. Spearman's correlation coefficients were calculated to test correlations between the FoP-Q-SF scores, the SF-12, and the HADS, and values higher than 0.50 indicated a strong correlation.

Internal consistency was examined using Cronbach's alpha and split-half correlations with Spearman-Brown's correction, where values above 0.7 were considered acceptable.

\section{Identification of Patient Clusters and Characteristics Associated with Fear of Progression}

A receiver operating characteristic (ROC) curve analysis was performed to identify the most appropriate cutoff value for high FoP, using the HADS anxiety subscale cutoff ( $\geq 11$ points) as the gold standard to detect moderate or severe FoP[19]. The sensitivity and specificity were calculated. Frequencies and percentages are used to describe the degree to which moderate to severe FoP was present in AP patients.

Univariate and multivariate logistic regression analyses were used to identify patient variables (including demographic, social and economic characteristics, disease status, anxiety/depression and quality of life level) independently associated with high FoP.

All statistical analyses were performed using SPSS (version 26), AMOS (version 22) and Graph Prism $8 . p$ values $<0.05$ were considered significant.

\section{Results}

The development study included 417 patients. They all provided informed consent, but one patient only completed part of the study. Patient characteristics are presented in Table 1.

\section{Development of the FoP-Q-SF}

The FoP-Q-SF questionnaire initially included 12 items. Experts adjusted the language of the items according to the disease characteristics of AP patients and the Chinese cultural background. We further interpreted "progress" in item 1 as "further deteriorate or recur". We changed "contracting" and "stranger" in items 6 and 7 to "inheriting" and "others", respectively. According to the qualitative results, the content of item 11 was changed to "I worry about being a burden to my family". Three questions $(\mathrm{Q} 6, \mathrm{Q7}, \mathrm{Q} 10)$ in the FoP-Q-SF were excluded according to the item analysis (Table 2).

\section{Validation}

Content validity. The experts agreed that the AP-FoP-Q-SF covers all the important areas.

Construct validity. Principal component analysis identified two dimensions, which accounted for $57.4 \%$ of the variance in item scores. The factor analysis showed that only one item (item 11) had a factor loading greater than 0.45 in both dimensions, while the other items were significant in a single dimension. The results of the EFA are summarized in Table 3. After discussion among experts in the group and review of the published literature[21], item 11 was classified as factor 1. CFA matched the data to two factorial structures. The patch models are shown in Figure 1. The goodness-of-fit indices of the 2-factor structural model are shown in Table 4 . Factors 1 and 2 were named physical health and social and family, respectively.

Criterion validity. AP-FoP-Q-SF total scores and the health physical subscores

were significantly correlated with HADS depression, HADS anxiety, and SF-12 quality of life (physical and mental component summaries). Social and family subscores were positively correlated with HADS depression scores and negatively correlated with mental quality scores. The results of the correlation analysis are shown in Table 5.

\section{Reliability}

Cronbach's alpha coefficient for the AP-FoP-Q-SF was 0.771 . The Guttman split-half correlation coefficient was calculated at 0.748. 
The total summary score on the AP-FoP-Q-SF was 23.506 ( \pm 6.581 SD, range 9-45). According to the ROC curve analysis, the best suited cutoff score for relevant FoP was 26 points, resulting in an area under the curve of 0.805 ( $95 \% \mathrm{Cl}$ : $0.747-0.863, p<0.001)$. This provided a sensitivity of $74.0 \%$ and a specificity of $72.8 \%$. A total of 147 patients (35.3\%) reported FoP at or above the cutoff value of 26 points. Multiple logistic regression analysis indicated that age, number of recurrences and HADS anxiety were independently associated with high FoP scores (Figure 2).

\section{Discussion}

The fear that the illness will progress is one of the main reasons for distress in patients with AP. This fear is real and independent, and we developed a specific instrument to measure it. In this study, we firstly developed the AP-FoP-Q-SF which demonstrated acceptable psychometric properties: high internal coherence, good discriminant validity, reliable measurement results and adequate stability. The AP-FoPQ-SF is short (9 items), quick to use, simple to score and may be a useful tool in both routine practice and clinical trials. Secondly, we found almost one-third (35.3\%) of evaluated patients had high fear scores, even though the disease is usually well managed, and these scores were associated with psychological distress. The fears identified in this study need to be addressed even in patients whose disease is acute.

The AP-FoP-Q-SF is the first validated, reliable, and clinically feasible questionnaire developed for patients with AP to evaluate a specific fear. The original FoP-Q-SF was used only for chronic diseases. After expert discussion and qualitative interviews, the contents of the questionnaire were adjusted. According to the questionnaire data of patients with AP in multiple centers, scientific statistical analysis was carried out to exclude irrelevant and unimportant questionnaire items, making it more suitable for AP patients. Therefore, the AP-FoP-Q-SF was validated as a newly developed instrument. Construct validity was identified by EFA, and the model was confirmed with CFA, concluding that physical health factors and social and family factors accounted for $57.4 \%$ of the total variance, which is considered a good result. A further test of criterion validity correlated the AP-FoP-Q-SF scores to the patients' nonspecific psychological distress, such as their HADS anxiety and depression scores, and quality of life scores. The significant correlation indicated that quality of life decreased with increasing AP-FoP-Q-SF total scores. We observed a moderate correlational association among FoP, anxiety and depression, indicating that total AP-FoP-Q-SF scores are related to the associated psychological burden. The correlation coefficient was similar to that in other research[19]. The reliability of the questionnaire is acceptable.

Three patient variables were also associated with high fear scores, namely, age, recurrence times and HADS anxiety. Our results showed that younger patients tended to report more fear. One possible reason is that they consider their disease to be more unexpected and experience higher levels of psychological distress. The other reason is that younger patients have more responsibilities in their family and society, so the disease makes them more fearful. Furthermore, an association was found between HADS anxiety and FoP. Researchers found that anxious patients spend greater time thinking about the risk of recurrence[22]. There is an interesting result in this article that the more recurrences there were, the higher the FoP scores of patients with AP. The clinical symptoms and recurrence rate in recurrent AP are greater than those in patients experiencing a first attack[23, 24]. Somatic cues trigger vulnerabilities and thereby elicit worry.

This study has certain limitations. Firstly, our sample was only from three tertiary centers and thus lacks representativeness and needs to be verified in more populations. Secondly, the scale dimensions were not extracted and determined after a qualitative interview, thereby lacking a theoretical basis. Thirdly, convenience sampling weakens the generalizability of the findings in our study. In addition, the cross-sectional study design did not enable us to assess test-retest reliability (i.e., stability over time). Hence, this aspect needs to be further evaluated.

\section{Conclusion}

The AP-FoP-Q-SF is the first valid and reliable, clinically feasible questionnaire for patients with AP. It assesses the most important aspects of FoP in AP patients, and the score reflects their quality of life. We recommend that it could be used in future research as a clinical instrument to evaluate and monitor FoP. The high FOP level of AP patients is related to age, recurrence times and anxiety, suggesting that these patients require more attention and close psychological guidance.

\section{Declarations}

\section{Ethics approval and consent to participate}

The study was reviewed by the Ethics Committee of Northern Jiangsu People's Hospital, Affiliated Hospital of Nanjing University Medical School (Ethics Number: 2021 ky188). And conforms to the ethical guidelines of the Declaration of Helsinki. Verbal consent, rather than written consent, was obtained from each patient to guarantee the interviewees' anonymity. 
Not applicable.

\section{Availability of data and materials}

The datasets generated and/or analysed during the current study are not publicly available because of licensed but are available from the corresponding author on reasonable request.

\section{Competing interests}

The authors declare they have no competing interests.

\section{Funding}

This research was funded by National Natural Science Foundation (No.82004291); Natural Science Foundation of Jiangsu Province (No. BK20190907) and Six Talent Peaks Project of Jiangsu Province (No.WSN-325).

\section{Authors' contributions}

WC participated in the conception and design of the study, and interpretation. SM and XY conducted data acquisition and performed the statistical analysis, and drafted the manuscript. SX, GL and WG helped to draft the manuscript and revising it critically for important intellectual content. All authors read and approved the final manuscript.

\section{Acknowledgments}

We would like to thank the patients who participated this study.

\section{References}

1. Petrov MS, Yadav D: Global epidemiology and holistic prevention of pancreatitis. Nature reviews Gastroenterology \& hepatology 2019 , 16(3):175-184.

2. Mokrowiecka A, Pinkowski D, Malecka-Panas E, Johnson CD: Clinical, emotional and social factors associated with quality of life in chronic pancreatitis. Pancreatology: official journal of the International Association of Pancreatology (IAP) [et al] 2010, 10(1):39-46.

3. MA S, YANG X, LIU Z, GAO Y, ZHANG C, CHEN W, LU G, GONG W: Negative Emotion in Patients with Acute Pancreatitis and Its Risk Factors:A Mixed Methods Systematic Review. Nursing Journal of Chinese People's Liberation Army 2021, 38(07):6-9+15.

4. Reja D, Weisberg I: Tu1602 ACUTE PANCREATITIS WITH COMORBID ANXIETY AND DEPRESSION IS ASSOCIATED WITH INCREASED 30DAY READMISSIONS, LENGTH OF STAY, AND COSTS OF HOSPITALIZATION: ANALYSIS OF A NATIONWIDE INPATIENT COHORT. Gastroenterology 2020, 158(6).

5. Bolourani S, Diao L, Thompson DA, Wang P, Coppa GF, Patel VM, Giangola MD: Risk Factors for Early Readmission After Acute Pancreatitis: Importance of Timely Interventions. The Journal of surgical research 2020, 252:96-106.

6. Pezzilli R, Morselli-Labate AM, Campana D, Casadei R, Brocchi E, Corinaldesi R: Evaluation of patient-reported outcome in subjects treated medically for acute pancreatitis: a follow-up study. Pancreatology: official journal of the International Association of Pancreatology (IAP) [et al] 2009, 9(4):375-382.

7. Liu J, Zhang B: The Lived Experience of Inpatients With Acute Recurrent Pancreatitis: A Qualitative Research Study From West China. Gastroenterology nursing: the official journal of the Society of Gastroenterology Nurses and Associates 2020, 43(3):249-257.

8. Herschbach P, Dinkel A: Fear of progression. Recent results in cancer research Fortschritte der Krebsforschung Progres dans les recherches sur le cancer 2014, 197:11-29.

9. Herschbach P, Berg P, Dankert A, Duran G, Engst-Hastreiter U, Waadt S, Keller M, Ukat R, Henrich G: Fear of progression in chronic diseases: Psychometric properties of the Fear of Progression Questionnaire. Journal of Psychosomatic Research 2005, 58(6):505-511.

10. Simard S, Savard J: Fear of Cancer Recurrence Inventory: development and initial validation of a multidimensional measure of fear of cancer recurrence. Supportive Care in Cancer 2008, 17(3):241.

11. Mehnert A, Herschbach P, Berg P, Henrich G, Koch U: [Fear of progression in breast cancer patients-validation of the short form of the Fear of Progression Questionnaire (FoP-Q-SF)]. Z Psychosom Med Psychother 2006, 52(3):274-288.

12. Banks PA, Bollen TL, Dervenis C, Gooszen HG, Johnson CD, Sarr MG, Tsiotos GG, Vege SS: Classification of acute pancreatitis-2012: revision of the Atlanta classification and definitions by international consensus. Gut 2013, 62(1):102-111. 
13. Mehnert A, Herschbach P, Berg P, Henrich G, Koch U: [Fear of progression in breast cancer patients-validation of the short form of the Fear of Progression Questionnaire (FoP-Q-SF)]. Z Psychosom Med Psychother 2006, 52(3):274-288.

14. Boije K, Drocic A, Engström M, Bjerså K: Patients' Perceptions of Experiences of Recovering From Acute Pancreatitis: An Interview Study. Gastroenterology nursing: the official journal of the Society of Gastroenterology Nurses and Associates 2019, 42(3):233-241.

15. Theunis J, Nordon C, Falissard B, Orri M, Mengeaud V, Misery L: Development and preliminary validation of the patient-reported Chronic Itch Burden Scale assessing health-related quality of life in chronic pruritus. The British journal of dermatology 2021.

16. Bergman L, Chaboyer W, Pettersson M, Ringdal M: Development and initial psychometric testing of the Intrahospital Transport Safety Scale in intensive care. BMJ open 2020, 10(10):e038424.

17. Streiner DL, Norman GR, Cairney J: Health measurement scales: a practical guide to their development and use: Oxford University Press, USA; 2015.

18. Li J, Wang J, Xie Y, Feng Z: Development and Validation of the Modified Patient-Reported Outcome Scale for Chronic Obstructive Pulmonary Disease (mCOPD-PRO). International journal of chronic obstructive pulmonary disease 2020, 15:661-669.

19. Wagner T, Augustin M, Blome C, Forschner A, Garbe C, Gutzmer R, Hauschild A, Heinzerling L, Livingstone E, Loquai C et al: Fear of cancer progression in patients with stage IA malignant melanoma. European journal of cancer care 2018, 27(5):e12901.

20. Hu Lt, Bentler PM: Cutoff criteria for fit indexes in covariance structure analysis: Conventional criteria versus new alternatives. Structural equation modeling: a multidisciplinary journal 1999, 6(1):1-55.

21. Wu Q, Ye Z, Li L, Liu P: Reliability and validity of Chinese version of Fear of Progression Questionnaire-Short Form for cancer patients. 2015, 50(12):1515-1519.

22. Yang Y, Sun H, Liu T, Zhang J, Wang H, Liang W, Chen Y, Zhang B: Factors associated with fear of progression in chinese cancer patients: sociodemographic, clinical and psychological variables. J Psychosom Res 2018, 114:18-24.

23. Pai CG, Kamath MG, Shetty MV, Kurien A: Continuing episodes of pain in recurrent acute pancreatitis: Prospective follow up on a standardised protocol with drugs and pancreatic endotherapy. World journal of gastroenterology 2017, 23(19):3538-3545.

24. Yu B, Li J, Li N, Zhu Y, Chen Y, He W, Lu N: Progression to recurrent acute pancreatitis after a first attack of acute pancreatitis in adults. Pancreatology: official journal of the International Association of Pancreatology (IAP) [et al] 2020, 20(7):1340-1346.

\section{Tables}

Table 1 Demographic Characteristics of the Included Patients $(N=417)$ 


\begin{tabular}{|c|c|c|}
\hline Characteristics & $N(\%)$ & Mean \pm S.D. \\
\hline Age (years) & & $51.56 \pm 14.30$ \\
\hline \multicolumn{3}{|l|}{ Sex } \\
\hline Female & $179(42.9 \%)$ & \\
\hline Male & $238(57.1 \%)$ & \\
\hline \multicolumn{3}{|l|}{ Education level } \\
\hline Primary and below & $145(34.8 \%)$ & \\
\hline Junior high & $127(30.5 \%)$ & \\
\hline Senior high & $105(25.2 \%)$ & \\
\hline College and above & $40(9.6 \%)$ & \\
\hline \multicolumn{3}{|l|}{ Marital status } \\
\hline Married & $391(93.8 \%)$ & \\
\hline Unmarried & $26(6.2 \%)$ & \\
\hline \multicolumn{3}{|l|}{ Profession } \\
\hline Farmer or worker & $185(44.4 \%)$ & \\
\hline Government or public institution employee & $51(12.2 \%)$ & \\
\hline Other & $181(43.3 \%)$ & \\
\hline \multicolumn{3}{|l|}{ Annual household income } \\
\hline$<40,000 \rrbracket$ & $32(7.7 \%)$ & \\
\hline $40,000-100,0008$ & $147(35.3 \%)$ & \\
\hline 100,000-200,000区 & $157(37.6 \%)$ & \\
\hline$>200,000 \rrbracket$ & $81(19.4 \%)$ & \\
\hline \multicolumn{3}{|l|}{ Homestyle } \\
\hline Lives alone & $31(7.4 \%)$ & \\
\hline With family & $371(89.0 \%)$ & \\
\hline Other & $15(3.6 \%)$ & \\
\hline Recurrence Times & & $0.70 \pm 1.33$ \\
\hline \multicolumn{3}{|l|}{ Etiology } \\
\hline Biliary & $223(53.5 \%)$ & \\
\hline Hyperlipidemia & $133(31.9 \%)$ & \\
\hline Alcoholism & $12(2.9 \%)$ & \\
\hline Other & $49(11.8 \%)$ & \\
\hline \multicolumn{3}{|l|}{ Severity classification } \\
\hline SAP & $32(7.7 \%)$ & \\
\hline MSAP & $330(79.1 \%)$ & \\
\hline MAP & $55(13.2 \%)$ & \\
\hline CTSI score & & $5.10 \pm 1.76$ \\
\hline SIRS score & & $0.86 \pm 0.92$ \\
\hline
\end{tabular}


Note: $\mathrm{CTSI}=$ Computed Tomography Severity Index; MAP=Mild Acute Pancreatitis; MSAP=Moderately Severe Acute Pancreatitis; SAP=Severe Acute Pancreatitis; SIRS= Systemic Inflammatory Response Syndrome

Table 2 Descriptive Statistics and Item Analysis for the AP-FoP-Q-SF ( $N=417)$

\begin{tabular}{|c|c|c|c|c|c|c|c|c|c|c|c|c|}
\hline \multirow{2}{*}{$\begin{array}{l}\text { Item } \\
\text { No. }\end{array}$} & \multirow{2}{*}{$\begin{array}{l}\text { Mean } \\
\text { (SD) }\end{array}$} & \multirow{2}{*}{$\begin{array}{l}\text { Floor } \\
\text { effect } \\
(\%)\end{array}$} & \multirow{2}{*}{$\begin{array}{l}\text { Ceiling } \\
\text { effect } \\
(\%)\end{array}$} & \multirow[t]{2}{*}{ Skewness } & \multirow{2}{*}{$\begin{array}{l}\text { Factor } \\
\text { loading }\end{array}$} & \multirow{2}{*}{$\begin{array}{l}\text { Item-total } \\
\text { correlation }\end{array}$} & \multirow{2}{*}{$\begin{array}{l}\text { Upper } \\
\text { Mean } \\
\text { (SD) }\end{array}$} & \multicolumn{3}{|l|}{ Lower } & \multirow{2}{*}{$\begin{array}{l}\text { Cronbach's a } \\
\text { if item } \\
\text { deleted }\end{array}$} & \multirow{2}{*}{$\begin{array}{l}\text { Item } \\
\text { exclusion } \\
\text { or } \\
\text { retention }\end{array}$} \\
\hline & & & & & & & & $\begin{array}{l}\text { Mean } \\
\text { (SD) }\end{array}$ & $\mathrm{T}$ & $P$ & & \\
\hline \multirow[t]{2}{*}{1} & 3.14 & 10.8 & 17.7 & -0.05 & 0.59 & 0.58 & 4.07 & 2.36 & 12.28 & 0.000 & 0.776 & Retained \\
\hline & (1.23) & & & & & & (1.02) & (1.12) & & & & \\
\hline \multirow[t]{2}{*}{2} & 2.33 & 32.1 & 7.2 & 0.59 & 0.58 & 0.57 & 3.19 & 1.46 & 12.36 & 0.000 & 0.777 & Retained \\
\hline & (1.20) & & & & & & (1.28) & $(0.80)$ & & & & \\
\hline \multirow[t]{2}{*}{3} & 3.22 & 8.4 & 20.4 & -0.01 & 0.51 & 0.51 & 4.08 & 2.43 & 12.10 & 0.000 & 0.784 & Retained \\
\hline & $(1.20)$ & & & & & & $(0.98)$ & (1.11) & & & & \\
\hline \multirow[t]{2}{*}{4} & 2.40 & 38.8 & 9.1 & 0.48 & 0.51 & 0.55 & 3.32 & 1.56 & 11.40 & 0.000 & 0.782 & Retained \\
\hline & (1.37) & & & & & & (1.42) & $(0.87)$ & & & & \\
\hline \multirow[t]{2}{*}{5} & 2.23 & 31.9 & 8.2 & 0.90 & 0.59 & 0.57 & 3.17 & 1.45 & 12.19 & 0.000 & 0.777 & Retained \\
\hline & (1.18) & & & & & & (1.31) & $(0.77)$ & & & & \\
\hline \multirow[t]{2}{*}{6} & 1.39 & 76.5 & 2.9 & 2.76 & 0.43 & 0.43 & 1.84 & 1.09 & 6.01 & 0.000 & 0.788 & Excluded \\
\hline & $(0.87)$ & & & & & & $(1.30)$ & $(0.34)$ & & & & \\
\hline \multirow[t]{2}{*}{7} & 1.79 & 52.5 & 4.3 & 1.46 & 0.55 & 0.54 & 2.51 & 1.31 & 8.41 & 0.000 & 0.780 & Excluded \\
\hline & (1.07) & & & & & & $(1.42)$ & $(0.59)$ & & & & \\
\hline \multirow[t]{2}{*}{8} & 2.17 & 34.3 & 5.0 & 0.79 & 0.50 & 0.51 & 2.80 & 1.55 & 8.79 & 0.000 & 0.783 & Retained \\
\hline & (1.11) & & & & & & $(1.26)$ & $(0.88)$ & & & & \\
\hline \multirow[t]{2}{*}{9} & 2.88 & 16.5 & 12.9 & 0.11 & 0.66 & 0.64 & 3.87 & 1.91 & 15.28 & 0.000 & 0.769 & Retained \\
\hline & $(1.22)$ & & & & & & (1.04) & $(0.93)$ & & & & \\
\hline \multirow[t]{2}{*}{10} & 2.03 & 40.3 & 2.4 & 0.77 & 0.44 & 0.46 & 2.47 & 1.48 & 7.22 & 0.000 & 0.787 & Excluded \\
\hline & (1.05) & & & & & & (1.22) & $(0.84)$ & & & & \\
\hline \multirow[t]{2}{*}{11} & 2.87 & 16.1 & 13.2 & 0.16 & 0.69 & 0.68 & 3.90 & 1.88 & 15.44 & 0.000 & 0.764 & Retained \\
\hline & (1.27) & & & & & & (1.07) & $(0.94)$ & & & & \\
\hline \multirow[t]{2}{*}{12} & 2.27 & 40.0 & 6.7 & 0.61 & 0.56 & 0.60 & 3.23 & 1.50 & 11.63 & 0.000 & 0.775 & Retained \\
\hline & (1.29) & & & & & & (1.36) & $(0.85)$ & & & & \\
\hline
\end{tabular}

Table 3 Results of Exploratory Factor Analysis $(N=122)$ 


\begin{tabular}{|lllc|}
\hline NO. & Item description & Factor 1 & Factor 2 \\
\hline 5 & When I am anxious, I have physical symptoms, e.g., rapid heartbeat, stomach ache, or nervousness. & 0.79 & 0.15 \\
\hline 9 & I am afraid of severe medical treatments in the course of my illness. & 0.73 & 0.25 \\
\hline 3 & I am afraid of pain. & 0.70 & 0.04 \\
\hline 2 & I am nervous prior to doctors' appointments or examinations. & 0.70 & -0.00 \\
\hline 1 & I become anxious if I think my disease may further deteriorate or recur. & 0.65 & 0.17 \\
\hline 11 & I worry about being a burden to my family. & 0.56 & 0.46 \\
\hline 4 & The thought that I might become less productive at my job upsets me. & -0.00 & 0.91 \\
\hline 12 & The thought that I might not be able to work due to my illness upsets me. & 0.09 & 0.91 \\
\hline 8 & I am worried I will no longer be able to pursue my hobbies because of my illness. & 0.25 & 0.51 \\
\hline
\end{tabular}

Table 4 Results of Confirmatory Factor Analysis ( $N=295)$

\begin{tabular}{|lllllll|}
\hline Fit indices & $\chi^{2} / d f$ & RMSEA & NFI & IFI & TLI & CFI \\
\hline Value & 2.803 & 0.078 & 0.903 & 0.935 & 0.909 & 0.934 \\
\hline
\end{tabular}

Note: $\chi^{2} / d f=$ chi squared/degrees of freedom, NFI=normed fit index, IFI=incremental fit index, TLI = Tucker-Lewis index, CFI = comparative fit index, and RMSEA = root mean square error of approximation.

Table 5 Association Between AP-FoP-Q-SF Scores and Validation Scale Scores $(N=416)$

\begin{tabular}{|lllll|}
\hline AP-FoP-Q-SF Scales & HADS anxiety & HADS depression & SF-12 physical score & SF-12 mental score \\
\hline Physical health & $0.6348^{\star *}$ & $0.494^{\star \star}$ & $-0.219^{\star *}$ & $-0.487^{\star \star}$ \\
\hline Social and family & $0.203^{\star \star}$ & 0.094 & 0.066 & $-0.133^{\star \star}$ \\
\hline Total score & $0.530^{\star \star}$ & $0.372^{\star \star}$ & $-0.096^{*}$ & $-0.395^{\star \star}$ \\
\hline
\end{tabular}

Note: $*=P<0.05, * *=P<0.001$

Figures 


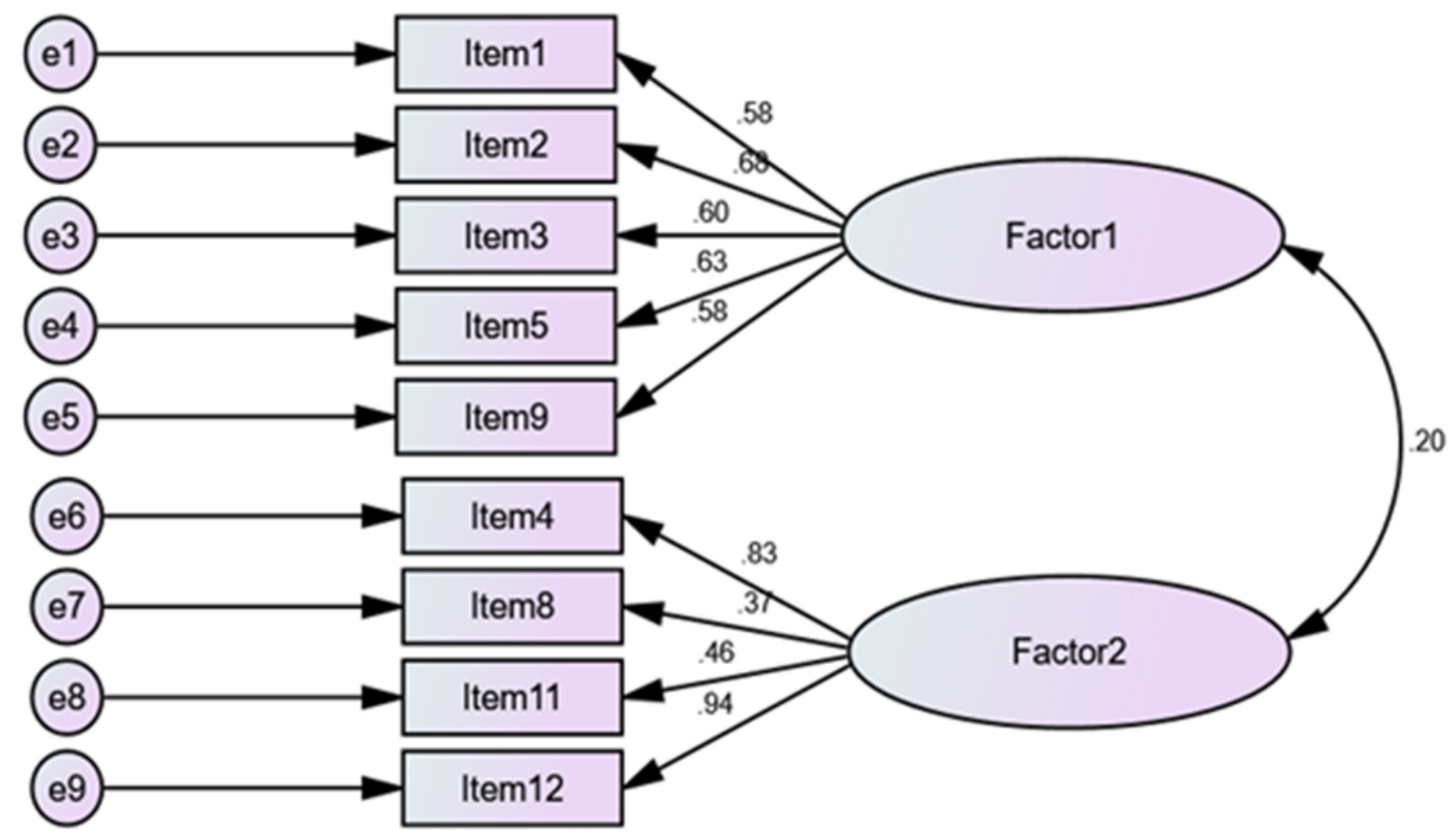

Figure 1

Confirmatory factor analysis model 


\section{Factors associated with FOP-Q-SF scores (multiple logistic regression)}

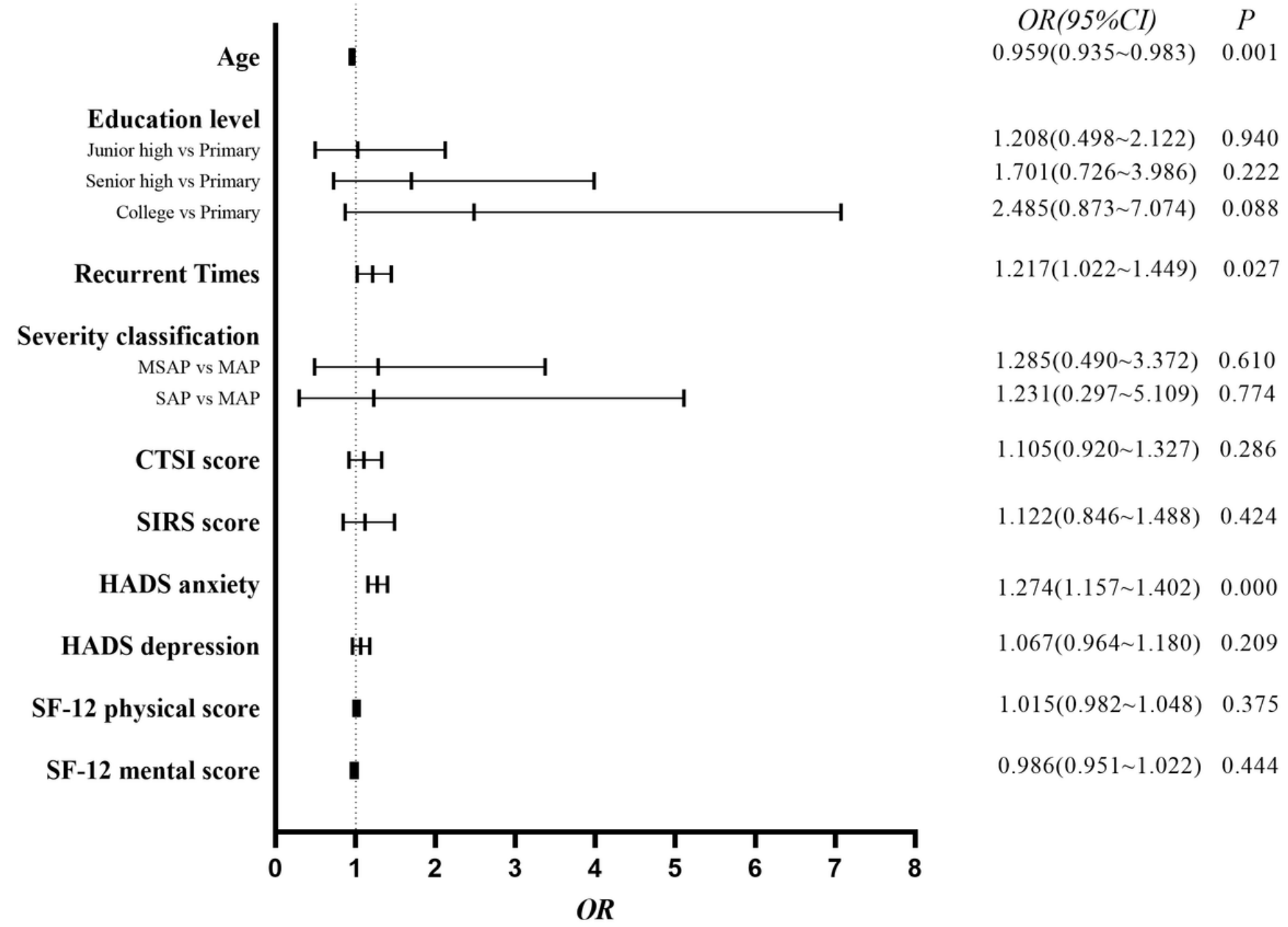

Figure 2

Variables independently associated with a high FoP score 